\title{
Cu ve Fe Metalleri için tiyadiazol türevi bileşiklerin kuantum kimyasal hesaplamaları ve korozyon inhibisyon aktiviteleri
}

\author{
Quantum chemical calculations and corrosion inhibition activities of thiadiazole derivative \\ compounds for $\mathrm{Cu}$ and Fe metals
}

\author{
Tuncay KARAKURT*1,a \\ ${ }^{I}$ Kırşehir Ahi Evran Üniversitesi, Mühendislik-Mimarlık Fakültesi, Kimya ve Proses Mühendisliği, Kırşehir
}

\begin{tabular}{llll}
\hline Geliş tarihi / Received: 22.01.2021 • Düzeltilerek geliş tarihi / Received in revised form: 25.03.2021 • Kabul tarihi / Accepted: 31.03 .2021 \\
\hline
\end{tabular}

\begin{abstract}
Öz
Bu çalışmada, Tiyadiazol türevi bileşiklerin (L1, L2, L3 ve L4) Cu ve Fe metal atomları üzerindeki korozyon inhibisyonu etkisini araştırmak için teoriksel hesaplama çalışmaları gerçekleştirilmiştir. Tüm bileşiklerin moleküler modellemeleri, Gaussian 09 programı ile Yoğunluk Fonksiyonel Teorisi DFT/B3LYP ve 6-311G (d) baz seti kullanılmıştır. İnhibitör adayı olan bileşiklerin inhibisyon etkinliği, $\mathrm{Fe}$ ve $\mathrm{Cu}$ metalleri için; $\mathrm{L} 3>\mathrm{L} 1>\mathrm{L} 4>\mathrm{L} 2$ sırası gözlendi. Tiyadiazol türevi bileşiklerin inhibe edici etkisinin, bu bileşiklerde bulunan fonksiyonel grupların elektronik doğasına bağlı olduğu görülmüştür. Tüm bileşiklerin kuantum kimyasal parametreleri olan; HOMO ve LUMO yörünge enerjileri, bazı kuantum kimyasal parametreleri; elektron afinitesi (EA), iyonizasyon potansiyeli (IP), elektronegatiflik $(\chi)$, mutlak sertlik $(\eta)$, mutlak yumuşaklık (S) ve kimyasal potansiyel $(\mu)$ değerleri hesaplandı. Bu parametreler kullanılarak, tüm bileşiklerin inhibitörden metale olan yük transferini ifade eden elektron transferlerinin kesri $(\Delta \mathrm{N})$ hesaplanmıştır. Son olarak bileşiklerin hesaplanan korozyonu önleme etkileri birbirleriyle karşılaştırıldı.
\end{abstract}

Anahtar kelimeler: Elektron transfer kesri, Gaussian 09, HOMO-LUMO, Korozyon inhibisyon etkisi, Yoğunluk fonksiyonel teorisi

\begin{abstract}
In this study, theoretical computational studies were carried out to investigate the corrosion inhibition effect of Thiadiazole derivative compounds on $\mathrm{Cu}$ and $\mathrm{Fe}$ metal atoms. Molecular modeling of all compounds was used with Gaussian 09 program, DFT/B3LYP theorem and 6-311G (d) base set. Inhibition activities of the compounds which are inhibitor candidate for $\mathrm{Fe}$ and $\mathrm{Cu}$ metals were observed in the order $\mathrm{L3}>\mathrm{L1}>\mathrm{L4}>\mathrm{L2}$. It has been found that the inhibitory effect of the Thiadiazole derivative compounds depends on the electronic nature of the functional groups present in these compounds. Quantum chemical parameters of all compounds; HOMO and LUMO orbital energies, some quantum chemical parameters; electron affinity (EA), ionization potential (IP), electronegativity ( $)$, global hardness ( $\eta)$, global softness $(S)$ and chemical potential $(\mu)$ values were calculated. Using these parameters, the electron transfers fraction $(\Delta N)$ of all compounds expressing the charge transfer from the inhibitor to the metal was calculated. Finally, the calculated anti-corrosion effects of the compounds were compared with each other.
\end{abstract}

Keywords: Electron transfer fraction, Gaussian 09, HOMO-LUMO, Corrosion inhibition effect, Density functional theory

*a Tuncay KARAKURT; tuncaykarakurt@gmail.com, Tel: (0386) 28060 62, orcid.org/0000-0001-6944-9883) 


\section{Giriş}

İnhibitörler, metallerin çözünme hızını azaltmak için çok etkilidir. Azot, kükürt ve oksijen içeren bileşikler inhibitör olarak rapor edilmiştir (Shukla ve Quraishi 2009; Singh vd., 2011; Athar vd., 2002; Quraishi ve Ansari, 2003; Quraishi ve Khan, 2006; Quraishi vd., 2008). Organik inhibitörler genellikle metal yüzeyinde bir film oluşturarak metali korozyondan korur. Bir molekülün inhibisyon etkinliği kimyasal bileşimine, moleküler yapısına ve metal yüzeye olan afinitesine bağlıdır. En etkili organik inhibitörler, yapılarında $\pi$ bağları olan organik bileşiklerdir. $\mathrm{Bu}$ bileşiklerin adsorpsiyonu, elektronik yapı, donör bölgesindeki sterik etki, aromatiklik, elektron yoğunluğu ve fonksiyonel gruplar $(-\mathrm{CHO},-\mathrm{N}=\mathrm{N}$, $\mathrm{R}-\mathrm{OH}$ vb.) gibi faktörlerden etkilenir (Singh ve Quraishi, 2010; Lukovits vd., 1995; Ayers Jr ve Hackerman, 1963; Shukla vd., 2008; Quraishi ve Shukla, 2009; Shukla vd., 2009). Deneysel yöntemler korozyon inhibisyonu mekanizmasını açıklamakta yararlıdır, ancak pahalı ve zaman alıcı oldukları için, korozyon inhibisyonu araştırmalarında teorik hesaplamalı kimya yöntemlerini kullanmak daha da önemli hale gelmektedir (Obi-Egbedi vd., 2011).

$\mathrm{Bu}$ çalışmada, literatürde daha önce sentezlenmiş olan Tiyadiazol türevi L1 (N-(5-(2-kloro-6florobenzil)-1,3,4-tiyadiazol-2-yl)benzamid), L2 (N-(5-(2-kloro-6- florobenzil)-1,3,4- tiyadiazol -2yl)-4-nitrobenzamid), L3 (N-(5-(2-kloro-6florobenzil)-1,3,4-tiyadiazol-2-yl)-4-

(metiltiyo)benzamid) ve L4 (3,4-dikloro-N-(5-(2kloro-6-florobenzil)-1,3,4-tiyadiazol-2-

yl)benzamid) dört adet bileşiğin (Er vd., 2018) temel durumdaki yapıları modellenerek moleküler geometrileri teorik yöntemlerle elde edildi. Bileşiklerin kararlı yapılarına ait inhibisyon aktiviteleri, $\mathrm{E}_{\text {Hомо, }} \mathrm{E}_{\mathrm{LUMO}}$, iyonizasyon potansiyeli (IP), elektron afinite (EA), mutlak yumuşaklık (S), kimyasal potansiyel $(\mu)$ ve mutlak elektronegatiflik $(\chi)$ gibi kuantum kimyasal tanımlayıcılarına bağımlı olarak hesaplanmıştır.

\section{Materyal ve metot}

\subsection{Teorik hesaplama yöntemleri}

Bileşiklerin moleküler modellemesinde, 6311G(d) (Foresman ve Frisch, 1996) baz setini kullanan yoğunluk fonksiyonel teorisi DFT/B3LYP (Hohenberg ve Kohn, 1964; Becke, 1992) ve PM3 (Stewart, 1989) yar1 deneysel metot kullanılmıştır. Tüm hesaplamalarda Gaussian 09 (Frisch vd., 2009) programı ve elde edilen sonuçları görselleştirmek için de GaussView 5 (Dennington vd., 2009) programı kullanılmıştır.

\subsection{PES analizi}

Tüm bileşiklerin kararlı yapıları, enerji yüzeyi taraması (PES) analizi ve yarı deneysel hesaplama metodu olan PM3 kullanılarak elde edilmiştir. Bu hesaplama bileşiklere ait $\theta 1(\mathrm{C} 3-\mathrm{C} 8-\mathrm{C} 9-\mathrm{S} 10)$ ve $\theta 2(\mathrm{C} 22-\mathrm{C} 17-\mathrm{C} 15-\mathrm{N} 14)$ dihedral açıları seçilerek yapılmıştır. Her bir açı $+180^{\circ} \% 180^{\circ}$ aralığında $20^{\circ}$ 'lik açılarla değiştirilerek her bir adımda tek nokta enerjileri hesaplanmıştır. PES analizi sonucu üç molekülde de $0^{\circ}$ de simetrik 4 minimum enerjiye karş1lık gelen konformasyonlar (c1-4) elde edildi (Şekil 1). 16 konformasyonun yeniden DFT/B3LYP/6-311G(d) yöntemi kullanılarak optimize işlemleri sonucunda, L1, L2, L3 ve L4 moleküllerine ait en kararlı yapılar elde edildi (Şekil 2). Tüm hesaplamalarda bu kararlı yapılar kullanılmıştır.

\section{Bulgular ve tartışma}

\subsection{Frontier orbitaller ve MEP haritası}

Elektronlarca işgal edilen en yüksek enerjiye sahip moleküler orbital HOMO ve işgal edilmemiş en düşük enerjiye sahip moleküler orbital ise LUMO olarak isimlendirilir. Bu orbitaller sınır moleküler orbital (FMO) olarak isimlendirilir. HOMO ve LUMO orbitalleri elektron verici ve alıc1 gruplar olarak da adlandırılabilir (Fukui, 1982; Buyukuslu vd., 2010). Moleküler aras1 yük transferlerinin belirlenmesinde kullanılan bu orbitaller, iyonlaşma potansiyeli, elektron afinitesi, kimyasal reaktivite, elektronegatiflik ve elektrofiliklik endeksi gibi moleküler özelliklerin hesaplanmasinda da kullanılır (Parr ve Pearson, 1983; Parr vd., 1978; Parr vd., 1999). Bileşiklerin HOMO ve LUMO orbitallerinin dağılımları Şekil 3'te gösterilmiştir. 


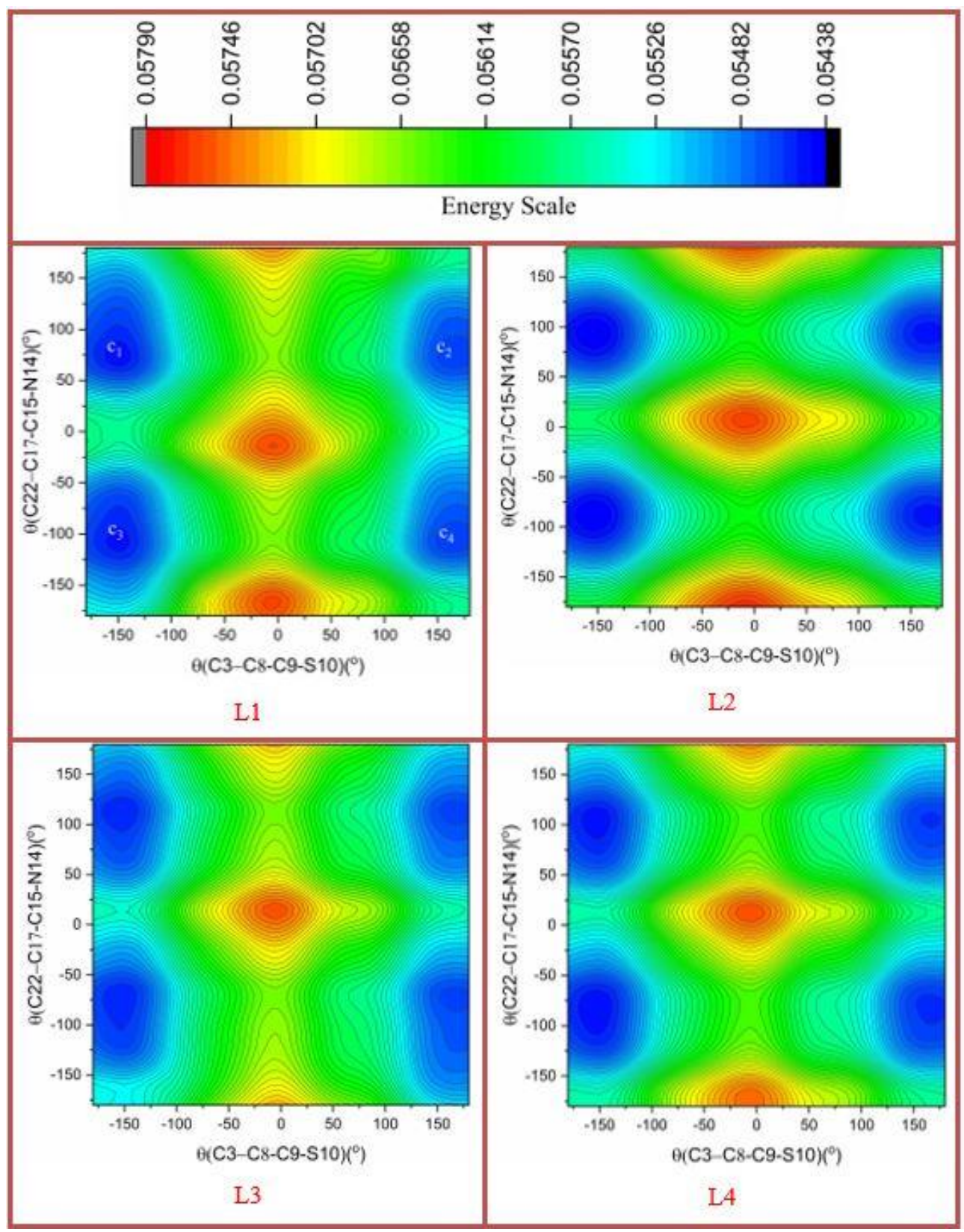

Şekil 1. Bileşiklerin 3D PES analizi haritası

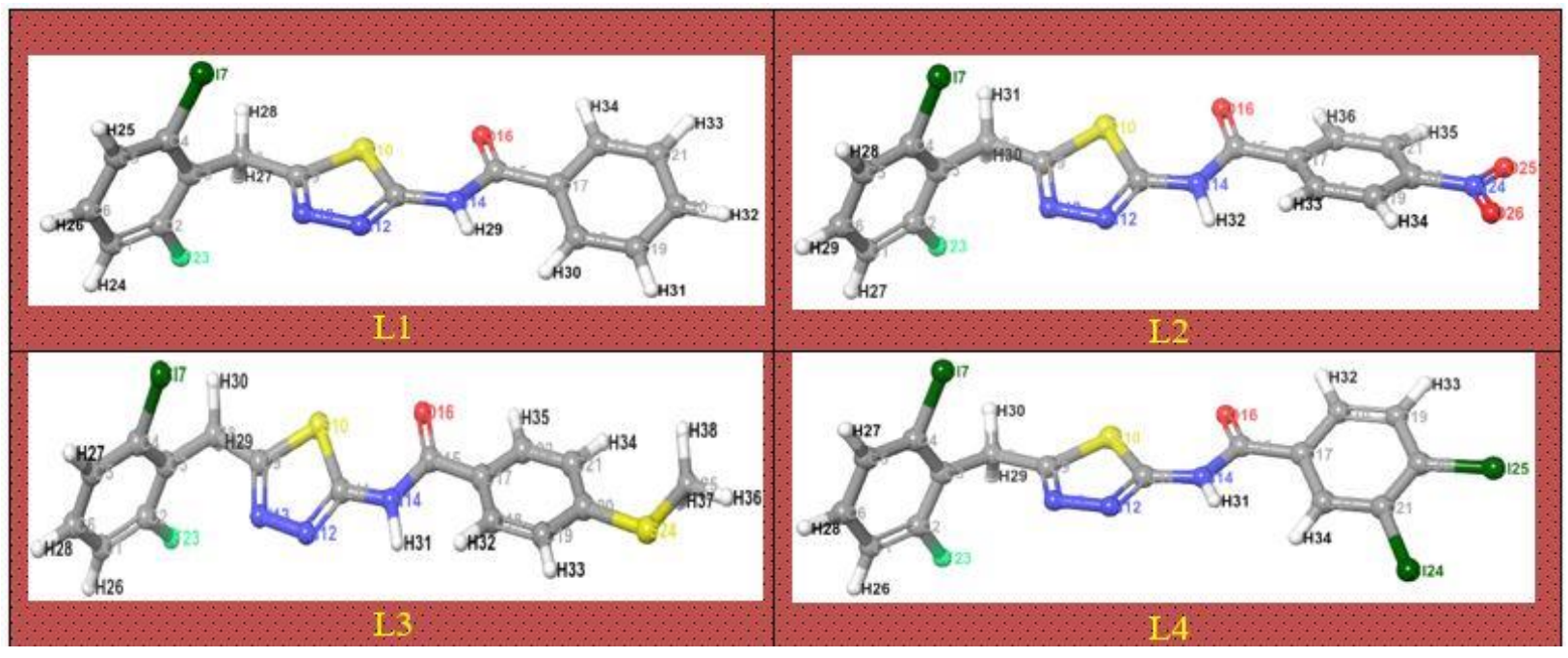

Şekil 2. Bileşiklerin kararlı yapıları 


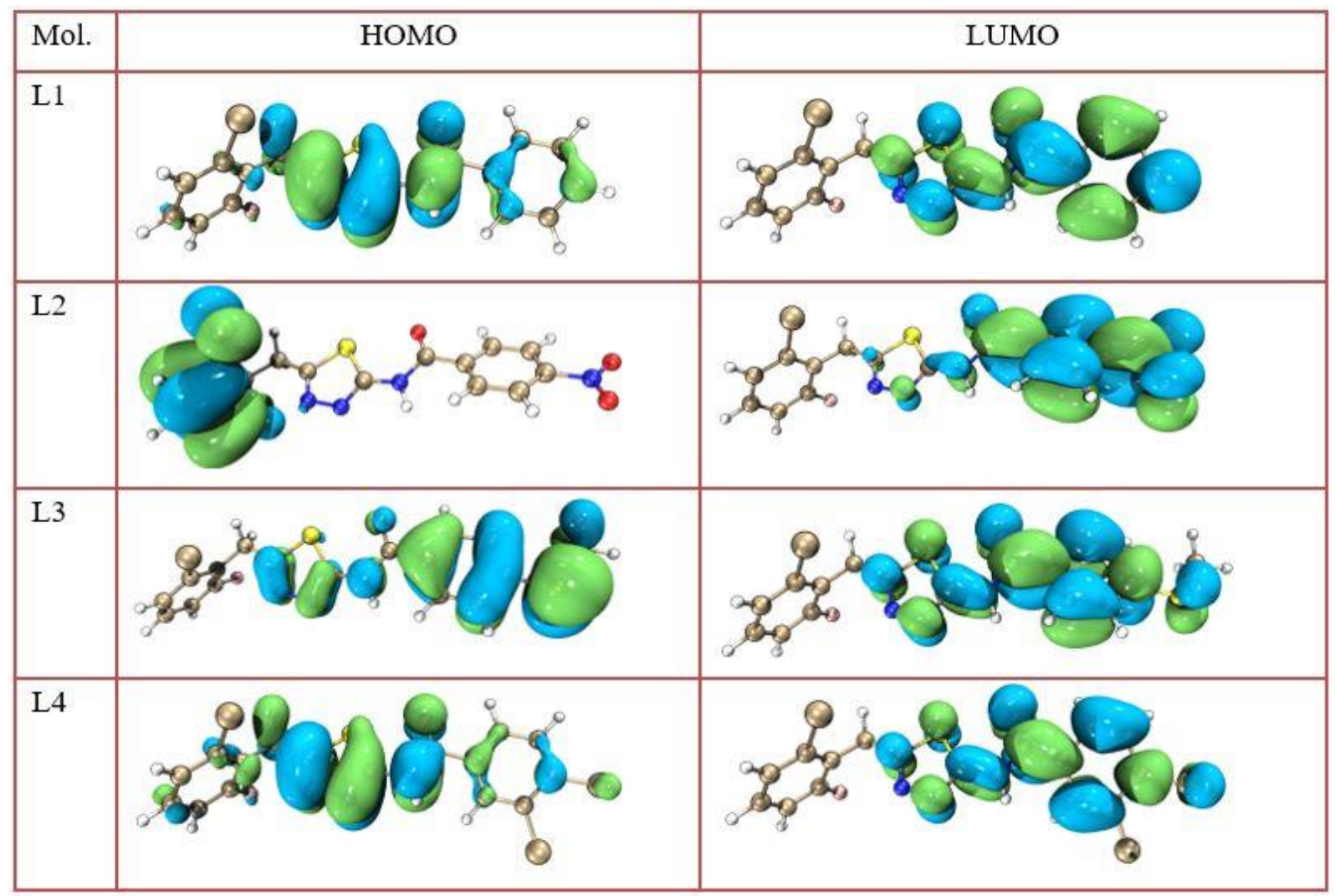

Şekil 3. Moleküllere ait Frontier orbitallerin gösterimi

$\mathrm{Bu}$ orbitallare en yüksek 5 atomun atomik katkısı; L1 bileşiği için LUMO: C15(\%16)+ O16(\%13)+ $\mathrm{C} 20(\% 12)+\mathrm{C} 18(\% 8)+\mathrm{C} 19(\% 4), \quad$ HOMO: $\mathrm{N} 12(\% 19)+\mathrm{N} 14(\% 17)+\mathrm{C} 9(\% 16)+\mathrm{N} 13(\% 12)+$ C11(\%12), L2 bileşiği için LUMO: N24(\%16)+ $\mathrm{O} 25(\% 15)+\mathrm{O} 24(\% 15)+\mathrm{C} 17(\% 10)+\mathrm{C} 20(\% 9)$, HOMO: $\mathrm{C} 5(\% 21)+\mathrm{C} 1(\% 20)+\mathrm{C} 2(\% 15)+$ $\mathrm{Cl}(\% 14)+\mathrm{F} 23(\% 7)$, L3 bileşiği için LUMO: $\mathrm{C} 15(\% 15)+\mathrm{O} 16(\% 13)+\mathrm{C} 20(\% 12)+\mathrm{C} 17(\% 11)+$ $\mathrm{C} 22(\% 8), \quad \mathrm{HOMO}: \quad \mathrm{S} 24(\% 42)+\mathrm{C} 17(\% 10)+$ $\mathrm{C} 20(\% 8)+\mathrm{C} 19(\% 7)+\mathrm{C} 21(\% 6)$ ve L4 bileşiği için LUMO: $\mathrm{N} 12(\% 18)+\mathrm{N} 14(\% 16)+\mathrm{C} 9(\% 16)+$ $\mathrm{N} 13(\% 12)+\mathrm{C} 11(\% 12), \quad \mathrm{HOMO}: \mathrm{C} 33(\% 12)+$ $\mathrm{N} 29(\% 11)+\mathrm{N} 25(\% 11)+\mathrm{C} 34(\% 10)+\mathrm{C} 39(\% 10)$ olarak hesaplanmıştır.

Durum Yoğunluğu (DOS), kat1 hal fiziği için önemli bir kavramdır, birim enerji aralığında durum sayısını temsil eder. Şekil 4'te molekülleri oluşturan s, p, d atomik orbitaller ve Toplam Durum Yoğunluğu (TDOS) gösterilmiştir. Şekilde atomik orbitallerin daha çok s ve p orbitallerden oluştuğu görülmektedir.

Moleküler elektrostatik potansiyeli (MEP) tanımlamak için renk kodlama sistemi kullanılır. MEP haritasından türetilmiş bileşiklerin moleküler yüzey haritalarına (Şekil 5) bakıldığında kırmızı renk kısmen negatif yük veya elektron bakımından zengin, mavi renk ise kısmen pozitif yük veya elektron eksikliği olan bölgelerini temsil eder. Şekil 5'te görüldüğü gibi moleküllerin en negatif bölgeleri, O16, O25, O26 N18, N12 ve N43 atomları etrafinda bulunulurken en pozitif bölgeler N14 atomuna bağlı $\mathrm{H}$ atomu ve halkalar üzerindeki $\mathrm{H}$ atomları üzerinde bulunmaktadır. Sonuç olarak, $\mathrm{O}$ ve $\mathrm{N}$ atomlarının herhangi bir ligant-reseptör etkileşimde diğer atomlara göre daha güçlü etkileşimler göstereceği söylenebilir. 


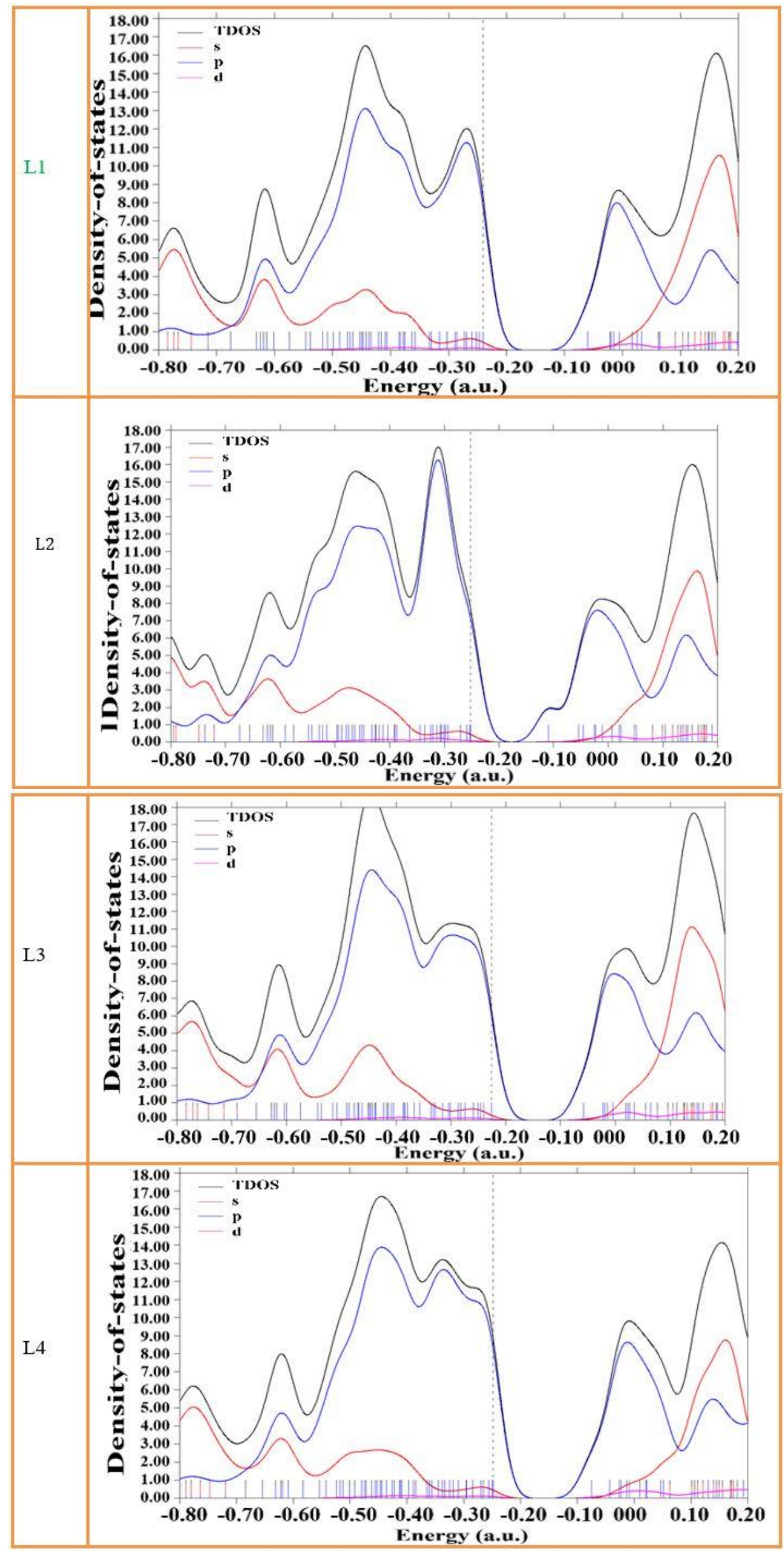

Şekil 4. Bileşiklerin TDOS, s, p ve d orbitalleri (Dikey kesik çizgiler, her bir bileşiğin HOMO enerji seviyesine karşıllk gelir) 


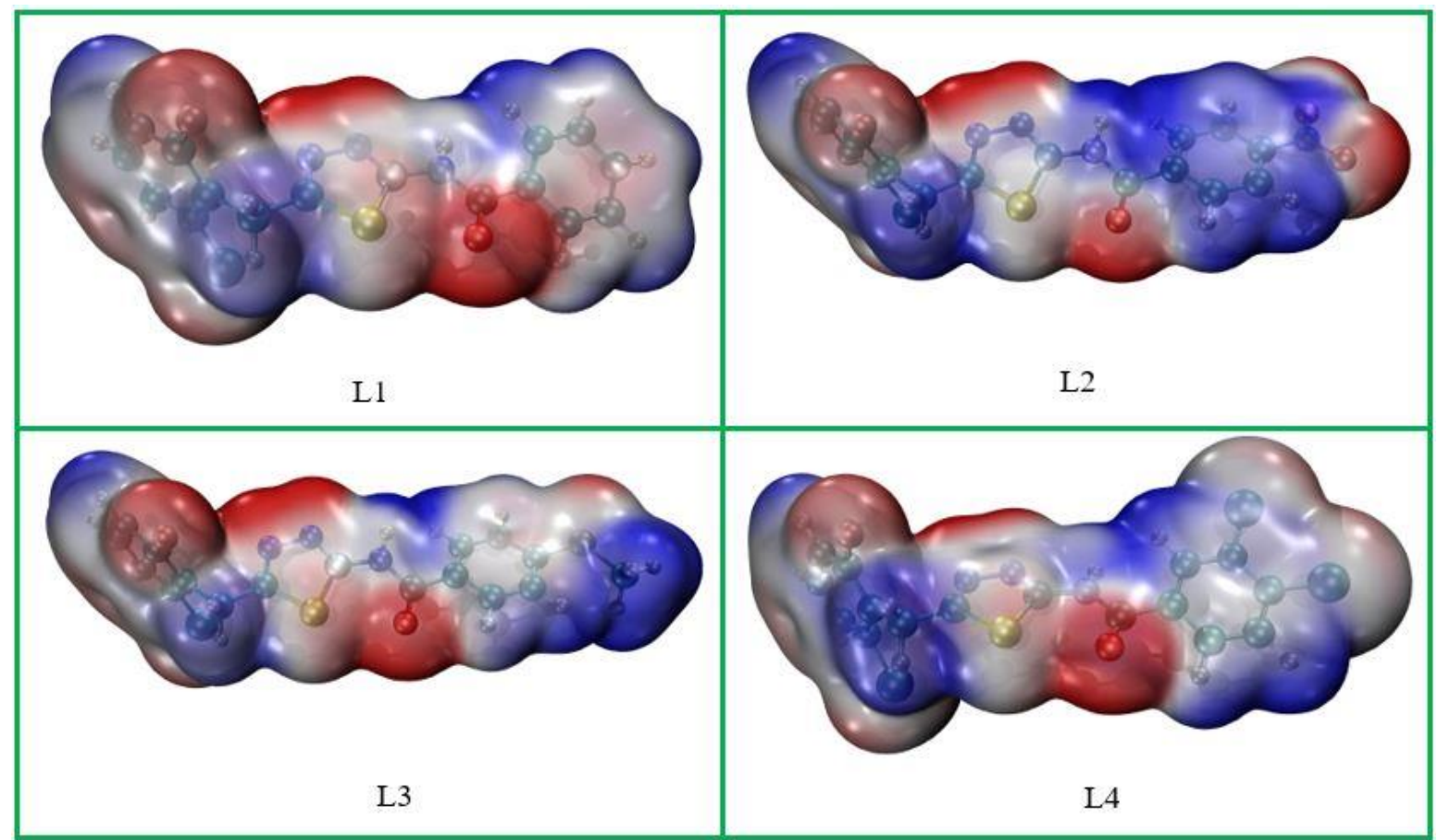

Şekil 5. Elektron yoğunluğundan türetilmiş MEP yüzeyleri

\subsection{Kimyasal Reaktivite Tanımlayıcıları}

Bir bileşiğin HOMO orbital enerjisi doğrudan iyonlaşma potansiyel enerjisi (IP) ile LUMO orbital enerjisi ise elektron afinitesi (EA) ile ilişkilidir ve aşağıdaki gibi verilir;

$\mathrm{IP}=-\mathrm{E}_{\text {HОмО }}$

$\mathrm{EA}=-\mathrm{E}_{\mathrm{LUMO}}$

Aynı şekilde elektronegativite $(\mathrm{X})$, mutlak sertlik $(\eta)$, kimyasal potansiyel $(\mu)$ ve global sertlik (S) indeksleri (Parr vd., 1978; Yang ve Parr, 1985) aşağıdaki denklemlerle verilir.

$\mathrm{X}=\mathrm{IP}+\mathrm{EA} / 2$

$\eta=I P-E A / 2$

$\mu=-x$

$\mathrm{S}=\mathrm{n} / 2$

Tablo 1'de tüm bileșiklere ait hesaplanan bu değerler verilmiştir. En kararlı yapı, toplam enerjisi -2733.53598 a.u. olan L4 bileşiğine aittir.

Tablo 1. Bileşiklerin kimyasal reaktivite belirleyicileri

\begin{tabular}{|c|c|c|c|c|c|c|c|c|}
\hline Mol. & Еномо (а.и.) $_{\text {. }}$ & $\boldsymbol{E}_{\text {LUMO }}($ a.u. $)$ & $\Delta E($ a.u. $)$ & $\eta($ a.u. $)$ & $S($ a.u. $)$ & $\mu($ a.u. $)$ & $x($ a.u. $)$ & $E_{t o p}($ a.u. $)$ \\
\hline L1 & -0.2486 & -0.06874 & -0.17986 & 0.08993 & 5.55988 & -0.15867 & 0.15867 & -1814.29705 \\
\hline $\mathbf{L} 2$ & -0.26081 & -0.11884 & -3.86323 & 0.070985 & 7.043742 & -0.18983 & 0.189825 & -2018.85112 \\
\hline $\mathbf{L 3}$ & -0.23286 & -0.06737 & -4.50325 & 0.082745 & 6.042661 & -0.15012 & 0.150115 & -2251.83174 \\
\hline L4 & -0.25655 & -0.08449 & -4.68203 & 0.08603 & 5.811926 & -0.17052 & 0.17052 & -2733.53598 \\
\hline
\end{tabular}

$\mathrm{Bu}$ parametreler, tüm bileşiklere ait elektron transfer kesri $(\Delta \mathrm{N})$ değerlerini elde etmek için kullanılmıştır. $\Delta \mathrm{N}$, inhibitörden metal yüzeye geçen elektronların sayısını ifade eder (Sulaiman ve Onawole, 2016) ve aşağıdaki denklem ile hesaplanır.

$\Delta \mathrm{N}=\frac{x_{B}-x_{A}}{2\left(\eta_{B}+\eta_{A}\right)}$
Denklemdeki, $X_{B}$ ve $\eta_{B}$ metal atomlara ait sirasıyla elektronegativite ve mutlak sertlik iken $X_{A}$ ve $\eta_{A}$ ise L1-L4 bileşiklerine ait değerlerdir. Eğer $\Delta \mathrm{N}<3.6$ ise metal yüzeyinde verilen elektronların artmasıyla birlikte korozyon önleme etkisi de artmaktadır (Lukovits vd., 2001). Bu çalışmada Cu ve Fe metal atomları üzerindeki korozyon etkisi incelenmiş olup, $X_{C u}=4.48 \mathrm{eV} / \mathrm{mol}$ (Pearson, 
1988), $\eta_{C u}=0 \mathrm{eV} / \mathrm{mol}$ (Martinez, 2003), $X_{F e}=$ $7 \mathrm{eV} / \mathrm{mol}$ ve $\eta_{F e}=0 \mathrm{eV} / \mathrm{mol}$ (Gece ve Bilgiç, 2010) alınarak hesaplamalar yapıldı.

Tablo 2'deki verilere göre tüm bileşiklerin Fe metal atomu için korozyon önleme etkisi $\mathrm{Cu}$ metaline göre daha fazla çıkarken, L3 bileşiği hem Fe hem de $\mathrm{Cu}$ metalleri için daha yüksek değerlere sahip olduğu görüldü. Elde edilen bu bulgular, $\mathrm{Fe}$ ve $\mathrm{Cu}$ metalleri için korozyon inhibitörü olarak L3 bileşiğinin diğer üç bileşiğe göre daha iyi sonuç vereceğini gösterir.

Tablo 2. Bileşiklerin inhibisyon değerleri

\begin{tabular}{|c|c|c|}
\hline İnhibitör & $\delta-\mathrm{Fe}$ & $\delta-\mathrm{Cu}$ \\
\hline $\mathrm{L} 1$ & 0.5481 & 0.0332 \\
\hline $\mathrm{L} 2$ & 0.4749 & -0.1774 \\
\hline L3 & $\mathbf{0 . 6 4 7 4}$ & $\mathbf{0 . 0 8 7 8}$ \\
\hline L4 & 0.5041 & -0.0342 \\
\hline
\end{tabular}

\section{Sonuçlar}

Literatürde sentezlenmiş olan Tiyadiazol türevi organik bileşik L1, L2, L3 ve L4'ün $\mathrm{Fe}$ ve $\mathrm{Cu}$ metalleri için korozyon inhibisyon etkinliği davranışını belirleyebilmek için, 6-311G(d) temel seti ile B3LYP fonksiyonel teoremi kullanılarak bir çalışma gerçekleştirilmiştir. Bileşiklerin hesaplanan HOMO ve LUMO enerjileri ile, elektron afinite (EA), iyonizasyon potansiyeli (IP), elektronegatiflik $(\chi)$, mutlak sertlik $(\eta)$, kimyasal potansiyel $(\mu)$, mutlak yumuşaklık (S) gibi bazı kuantum kimyasal parametreleri hesaplandi. Bu parametreler kullanılarak, tüm bileşiklerin inhibitörden metale olan yük transferini belirleyen elektron transfer kesri $(\Delta \mathrm{N})$ katsayıları hesaplandı. $\mathrm{SCH}_{3}$ fonksiyonel grubu bağlı olan L3 bileşiğinin hem $\mathrm{Fe}$ hem de $\mathrm{Cu}$ metalleri için en aktif bileşik olurken en az etki ise $\mathrm{NO}_{2}$ fonksiyonel grubu bağlı olan L2 bileşiğinde görülmüştür.

\section{Kaynaklar}

Athar, M., Ali, H. and Quraishi M. (2002). Corrosion inhibition of carbon steel in hydrochloric acid by organic compounds containing heteroatoms. British Corrosion Journal, 37 (2), 155-158. https://doi.org/10.1179/000705902225002376

Ayers, Jr R.C. and Hackerman, N. (1963). Corrosion inhibition in $\mathrm{HCl}$ using methyl pyridines. Journal of the Electrochemical Society, 110 (6), 507. https://doi.org/10.1149/1.2425802

Becke, A.D. (1992). Density-functional thermochemistry. I. The effect of the exchangeonly gradient correction. The Journal of chemical
physics,
96
(3),
2155-2160.
https://doi.org/10.1063/1.462066

Buyukuslu, H., Akdogan, M., Yildirim, G. and Parlak, C. (2010). Ab initio Hartree-Fock and density functional theory study on characterization of 3(5-methylthiazol-2-yldiazenyl)-2-phenyl-1Hindole. Spectrochimica Acta Part A: Molecular and Biomolecular Spectroscopy, 75 (4), 13621369. https://doi.org/10.1016/j.saa.2010.01.003

Dennington, R., Keith T. and Millam, J. (2009). GaussView, version 5.

Er, M., Abounakhla, A.M., Tahtaci, H., Bawah, A.H., Çınaroğlu, S.S., Onaran, A. and Ece, A. (2018). An integrated approach towards the development of novel antifungal agents containing thiadiazole: synthesis and a combined similarity search, homology modelling, molecular dynamics and molecular docking study. Chemistry Central Journal, $\quad 12 \quad(1), \quad 121$. https://doi.org/10.1186/s13065-018-0485-3

Foresman, J. and Frisch, A. (1996). Exploring chemistry with electronic structure methods: A guide to using Gaussian, Pittsburgh, PA: Gaussian. Inc,

Frisch, M., Trucks, G., Schlegel, H.B., Scuseria, G.E., Robb, M.A., Cheeseman, J.R., Scalmani, G., Barone, V., Mennucci, B. and Petersson, G. (2009). Gaussian 09, Revision d. 01, Gaussian. Inc, Wallingford CT, 201

Fukui, K. (1982). Role of frontier orbitals in chemical reactions. Science, 218 (4574), 747-754

Gece, G. and Bilgiç, S. (2010). A theoretical study of some hydroxamic acids as corrosion inhibitors for carbon steel. Corrosion science, 52 (10), 3304-3308.

https://doi.org/10.1016/j.corsci.2010.06.005

Hohenberg, P. and Kohn, W. (1964). Inhomogeneous electron gas. Physical review, 136 (3B), B864. ttps://doi.org/10.1103/PhysRev.136.B864

Lukovits, I., Kalman, E. and Palinkas, G. (1995). Nonlinear group-contribution models of corrosion inhibition. Corrosion, 51 (3), 201-205. https://doi.org/10.5006/1.3294362

Lukovits, I., Kalman, E. and Zucchi, F. (2001). Corrosion inhibitors-correlation between electronic structure and efficiency. Corrosion, 57 (1), 3-8. https://doi.org/10.5006/1.3290328

Martinez, S. (2003). Inhibitory mechanism of mimosa tannin using molecular modeling and substitutional adsorption isotherms. Materials Chemistry and Physics, 77 (1), 97-102. https://doi.org/10.1016/S0254-0584(01)00569-7 
Obi-Egbedi, N., Obot, I., El-Khaiary, M., Umoren, S. and Ebenso, E. (2011). Computational simulation and statistical analysis on the relationship between corrosion inhibition efficiency and molecular structure of some phenanthroline derivatives on mild steel surface. Int J Electrochem Sci, 6 (5649), e5675

Parr, R.G., Donnelly, R.A., Levy, M. and Palke, W.E. (1978). Electronegativity: the density functional viewpoint. The Journal of Chemical Physics, 68 (8), 3801-3807. https://doi.org/10.1063/1.436185

Parr, R.G. and Pearson, R.G. (1983). Absolute hardness: companion parameter to absolute electronegativity. Journal of the American chemical society, 105 (26), 7512-7516. https://doi.org/10.1021/ja00364a005

Parr, R.G., Szentpaly L.V. and Liu, S. (1999). Electrophilicity index. Journal of the American Chemical Society, $121 \quad$ (9),1922-1924. https://doi.org/10.1021/ja983494x

Pearson, R.G. (1988). Absolute electronegativity and hardness: application to inorganic chemistry. Inorganic chemistry, 27 (4), 734-740. https://doi.org/10.1021/ic00277a030

Quraishi, M., Ahamad, I., Singh, A.K., Shukla, S.K., Lal, B. and Singh, V. (2008). N(Piperidinomethyl)-3-[(pyridylidene) amino] isatin: A new and effective acid corrosion inhibitor for mild steel. Materials chemistry and physics, $112 \quad$ (3), 1035-1039. https://doi.org/10.1016/j.matchemphys.2008.07. 011

Quraishi, M. and Ansari, F. (2003). Corrosion inhibition by fatty acid triazoles for mild steel in formic acid. Journal of applied electrochemistry, 33 (34), 233-238. https://doi.org/10.1023/A:1024106123577

Quraishi, M. and Khan, S. (2006). Inhibition of mild steel corrosion in sulfuric acid solution by thiadiazoles. Journal of applied electrochemistry, 36 (5), 539-544. https://doi.org/10.1007/s10800005-9087-6

Quraishi, M. and Shukla, S.K. (2009). Poly (anilineformaldehyde): a new and effective corrosion inhibitor for mild steel in hydrochloric acid. Materials Chemistry and Physics, 113 (2-3), 685-
689.

https://doi.org/10.1016/j.matchemphys.2008.08. 028

Shukla, S.K. and Quraishi, M. (2009). Ceftriaxone: a novel corrosion inhibitor for mild steel in hydrochloric acid. Journal of Applied

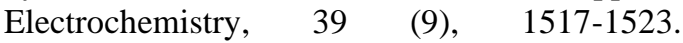
https://doi.org/10.1007/s10800-009-9834-1

Shukla, S.K., Quraishi, M. and Prakash, R. (2008). A self-doped conducting polymer "polyanthranilic acid": An efficient corrosion inhibitor for mild steel in acidic solution. Corrosion Science, 50 (10), 2867-2872. https://doi.org/10.1016/j.corsci.2008.07.025

Shukla, S.K., Singh, A.K., Ahamad, I. and Quraishi, M. (2009). Streptomycin: A commercially available drug as corrosion inhibitor for mild steel in hydrochloric acid solution. Materials Letters, 63 (9-10), 819-822. https://doi.org/10.1016/j.matlet.2009.01.020

Singh, A.K. and Quraishi, M. (2010). Effect of Cefazolin on the corrosion of mild steel in $\mathrm{HCl}$ solution. Corrosion Science, 52 (1), 152-160. https://doi.org/10.1016/j.corsci.2009.08.050

Singh, A.K., Shukla, S.K., Singh, M. and Quraishi, M. (2011). Inhibitive effect of ceftazidime on corrosion of mild steel in hydrochloric acid solution. Materials Chemistry and Physics, 129 (1-2), 68-76. https://doi.org/10.1016/j.matchemphys.2011.03. 054

Stewart, J.J. (1989). Optimization of parameters for semiempirical methods II. Applications. Journal of computational chemistry, 10 (2), 221-264. https://doi.org/10.1002/jcc.540100209

Sulaiman, K.O. and Onawole, A.T. (2016). Quantum chemical evaluation of the corrosion inhibition of novel aromatic hydrazide derivatives on mild steel in hydrochloric acid. Computational and Theoretical Chemistry, 1093, 73-80. https://doi.org/10.1016/j.comptc.2016.08.014

Yang, W. and Parr, R.G. (1985). Hardness, softness, and the fukui function in the electronic theory of metals and catalysis. Proceedings of the National Academy of Sciences, 82 (20), 6723-6726. https://doi.org/10.1073/pnas.82.20.6723 